\title{
Representaciones, Actitudes y Prácticas Respecto a la Leishmaniasis Cutánea en la Población del Cantón de Acosta, Provincia de San José, Costa Rica. Estudio Antropológico Exploratório'
}

\section{Representation, Attitudes, and Practices Related to Cutaneous Leishmaniasis in People from Acosta Country, San Jose Province, Costa Rica. An Exploratory Anthropological Astudy}

\section{Alvaro Dobles-Ulloa' \& Catherine Perriard}

\section{$D O B L E S-U L L O A, A$. $\mathcal{F} P E R R I A R D, C$. Representations, Attitudes, and Practices Related} to Cutaneous Leishmaniasis inPeople from Acosta Country, San Jose Province, Costa Rica. An Exploratoray Anthropological Study. Cad. Saúde Públ., Rio de Janeiro, 10 (2): 181-189, Apr/Jun, 1994.

An exploratory anthropological study on representations, attitudes, and practices related to Cutaneous Leishmaniasis (CL) among the rural population of Acosta Vountry, Costa Rica, aimed to estimate the applicability of epidemiologically-based control measures. Open interviews with a small sample of individuals from both case and control households provided the basic for a Propositional Discourse Analysis (PDA). Results are that Acosta people consider CL a distinct nosologic entity, but they are mainly interested in its clinical manifestations in children (who are mainly affected), as well as in their own capacity to act on the disease using folk remedies. The idea of control measures on reservoirs, on vectors, or on the spatial and temporal context of contact does not arise spontaneously in people's thinking. Nevertheless, CL is perceived as a disruption in the safe domiciliary and peridomiciliary space, so that control measures intervening there could have a chance for success.

Key words: Leishmaniasis; Anthropology; Control; Treatment; Epidemiology

\section{INTRODUCCION}

El problema planteado fue el de conocer las representaciones, actitudes y prácticas sobre la Leishmaniasis Cutánea en el cantón de Acosta,

\footnotetext{
${ }^{1}$ Este estudio fue posible gracias al Programa para Investigaciones en Aspectos Económicos y sociales de las Enfermedades Tropicales financiado por el Programa Especial de Investigación y Entrenamiento en Enfermedades Tropicales (TDR), Programa de las Naciones Unidas para el Desarrollo, Banco Mundial, Organización Mundial de la Salud, mediante convenio con el Laboratorio de Investigaciones Sociales de la Universidad Central de Venezuela. Proyecto SG-P90.68. ${ }^{2}$ Unidad de Epidemiología, Programa de Investigación en Enfermedades Tropicales, Facultad de Ciencias de la Salud Universidad Nacional "Campus Omar Dengo". Apartado Postal: 304-3000, Heredia, Costa Rica.
}

provincia de San José (Costa Rica), desde el punto de vista de la población afectada o en riesgo de serlo.

En aquella región rural, de acceso relativamente difícil, predomina una población agricultora, en general de escasos recursos económicos. La incidencia de Leishmaniasis Cutánea es alta (los registros de 1990 a 1992 y los estimados de población para esos años arrojan una tasa acumulada muy cercana a $9 /$ 1000).

Esta incidencia se ha venido concentrando en niños menores de 9 años (19/1000, según los mismos registros y estimados para ese grupo de edad), así como en mujeres adultas. Este cambio de situación apunta hacia una probable transmisión peridomiciliaria y aun intradomiciliaria de dicha enfermedad (Rojas et al., 1988). Ambos grupos salen raras veces al bosque (re- 
gistros no publicados de observación de conducta, conservados en la Unidad de Epidemiología de la Universidad Nacional).

Otros indicadores en esta dirección son la deforestación agravada desde hace más de veinte años, particularmente en los alrededores de las viviendas, y la captura repetida de hembras de Lutzomyia grávidas y con ingesta reciente de sangre dentro de laas viviendas (Murillo \& Zeledón, 1985).

La intención prátctica del studio fue la de obtener información cualitativa que permitiera estimar la viabilidad de aplicación (eventual recepción, aplicabilidad e impacto) de algunas medidas de control, propuestas basándose en el estudio epidemiológico cuantitativo de factores de riesgo (Rojas, 1992).

Se efectúo un estudio de carácter exploratorio, propiamente antropológico, fundamentalmente cualitativo pero basado en un tratamiento cuantitativamente controlado.

\section{METODO}

Se utilizó un diseño cualitativo, completo en sí mismo pero complementario del diseño cuantitativo de la investigación epidemiológica, respecto a la cual no se considera este estudio en ninguna manera inferior en rigor ni en carácter científico.

La muestra se extrajo de la lista de casas en que se había realizado encuesta epidemiológica, sea por haber presentado uno o más de sus habitantes como caso incidente de leishmaniasis cutánea o como control.

Primero se clasificaron las localidades en que hasta entonces había trabajado el proyecto epidemiológico, con arreglo a información estadística (Nuhn, 1978), actualizada según nuestras propias observaciones de campo, y según tres criterios socioeconómicos, a saber: uso agropecuario del suelo, ingreso agrícola, tamaño de las fincas.

Resultaron tres agrupaciones suficientemente homogéneas, que además se distribuyeron bastante continuamente en el espacio geográfico. Se resolvió distribuir equitativamente las casas previstas entre esas tres zonas, previendo que podría haber diferencias entre ellas; sin embargo posteriormente se comprobó que las entrevistas fueron bastante homogéneas y has diferencias entre ellas no correspondieron a la distinción de zonas propuesta.

Se usó una tabla de números al azar para extraer la muestra de los registros de casas (por entonces se hizo el corte a 170 casas ya participantes en el estudio epidemiológico), en el orden en que fueron saliendo. Sin embargo, atendiendo la sugerencia del epidemiólogo Julio César Rojas, el procedimiento se repitió cuando fue necesario para que al menos una de las casas en cada zona representara un caso incidente de Leishmaniasis Cutánea.

A la vista de los factores metodológicos mencionados, $\mathrm{y}$ teniendo en cuenta las limitaciones logísticas, el diseño previó 48 entrevistas, 4 en cada una de las 12 diferentes casas escogidas; se completaron todas, más 2 de prueba (para un total de 50).

Se considera que, en el diseño retenido, la profundidad compensa la extensión (Devereux, 1970). Se estima pues que las generalizaciones empíricas del estudio exploratorio se pueden aplicar a toda la población adulta del cantón de Acosta.

En cada una de 12 casas se efectuaron, como quedó dicho, cuatro entrevistas abiertas en tono de conversación, a intervalos espaciados (tres con la señora de la casa y una con el marido). Se favoreció el contacto interpersonal repetido, prolongado y profundamente humano, del que se obtuvieron "insights" importantes sobre sus representaciones, actitudes y prácticas relacionadas con la Leishmaniasis Cutánea y su tratamiento.

La consigna de la primera ronda de entrevistas integró el tema más general: concepciones, actitudes y prácticas respecto a la Leishmaniasis Cutánea. Las consignas de las rondas subsiguientes correspondieron a aspectos específicos, de importancia para los investigadores: percepción y uso del espacio doméstico y peridoméstico; percepción y uso de animales y plantas en ese espacio doméstico y peridoméstico; aspectos de la vivencia cotidiana de las mujeres y sus niños de hasta 9 años de edad que podrían estar relacionados con la transmisión del parásito; periodicidad de varios de esos aspectos en relación con ciclos más o menos largos, que son sociales en todo o en parte. 
El tratamiento cualitativo incluyó todas las entrevistas, pero en el tratamiento cuantitativo sólo se tornó en cuenta la primera ronda, para evitar sesgar la representación que los sujetos tienen sobre la Leishmaniasis Cutánea, que es de lo que se trata en el presente artículo.

El discurso de las personas entrevistadas respecto al tema de la Leishmaniasis Cutánea se produjo en condiciones relativamente controladas de entrevista "abierta", que al mismo tiempo fueron condiciones relativamente naturales de conversación, dentro del contexto social de los sujetos y en sus propias casas, con una dialogicidad libre para ellos, no directiva ni jerarquizada por parte de los entrevistadores. Todas las entrevistas tuvieron una extensión suficiente (unos 45 minutos en promedio), y fueron grabadas con la anuencia de la persona entrevistada; la mitad de ellas se consignó verbatim en trascripción posterior.

Las técnicas de entrevista se limitaron en principio a las llamadas técnicas reflexivas, a saber: el entrevistador retomó, sin cambiar nada, frases o partes limitadas del discurso del entrevistado; el entrevistador reenvió en eco la última palabra o el último sintagma pronunciado por el entrevistador; el entrevistador hizo reescuchar al sujeto todo o parte de su producción discursiva precedente, para incitarlo a producir un discurso complementario (Ghiglione et al., 1985A).

Se recurrió también como técnica al silencio de parte del entrevistador, acompañándolo de una actitud y gestualidad que transmitieron al entrevistado, sin presionarlo demasiado, que se tenía la seguridad de que diría más en un plazo breve.

Por supuesto, fue casi inevitable recurrir ocasionalmente a técnicas no reflexivas, dentro del carácter de coemisión que el sujeto puede darle a una entrevista de este tipo (se dieron casos en que fue la persona entrevistada la que comenzó la entrevista, y haciendo una pregunta directa).

$\mathrm{El}$ instrumento empleado para generar el tipo de discurso requerido por los objetivos, y este tipo de discurso mismo, suponen una limitación de la escala utilizable. Por el carácter del estudio, que no se propuso contrastar hipótesis sino explorar un campo desconocido y hacerlo en los términos del sujeto, una escala nominal se consideró suficiente.
Se efectuó un análisis proposicional del discurso, APD (Ghiglione et al., 1985): se cortaron las superficies textuales transcritas en unidades sintagmáticas menores, para cada una de las cuales se pudo identificar entre uno y cuatro referentes nucleares, sustantivos o equivalentes en posición de actantes. Se establecieron las equivalencias paradigmáticas para cada uno de estos referentes y se hizo el conteo de las unidades sintagmáticas que estructuraban, y del total de esas unidades en cada entrevista.

Por las razones explicadas más arriba, para el análisis cuantitativo del discurso se retuvieron las doce entrevistas de la primera ronda; las frecuencias relativas de los referentes se establecieron sobre la suma de las unidades sintagmáticas de cada una de esas doce entrevistas. Se retuvieron para el análisis ulterior los referentes cuya frecuencia relativa sobre el total de las doce entrevistas fue de $5 \%$ o mayor (límite arbitrario, que pretende garantizar la pertinencia informativa de los referentes escogidos).

Los referentes que llenaron esta exigencia se utilizaron para evaluar la homogeneidad de los discursos producidos; no se encontraron diferencias significativas en los patrones de respuesta.

De estos referentes, se escogieron aquellos que aparecieron en al menos once de las doce entrevistas (referentes nucleares), para estudiar sus coapariciones en el seno de una misma unidad sintagmática, en cada una de las entrevistas consideradas. La base para las frecuencias relativas fue la suma de las unidades sintagmáticas de todas las entrevistas, que asociaron dos o más de los referentes escogidos (se eliminaron aquellas estructuras por un solo referente).

El anterior análisis cuantitativo jerarquizó y reagrupó los referentes, permitiendo así orientar y controlar el subsecuente análisis cualitativo. Este último incorpora además, subordinadamente, otros elementos que son de importancia para la investigación y para el control de la Leishmaniasis Cutánea, pero que no aparecieron integrados en el marco cognitivo, afectivo ni pragmático de los sujetos.

Este estudio procura rendir el fondo de lo que la Leishmaniasis Cutánea significa en teoría, en sentimiento y en práctica para una población que se ve afectada por ella cotidianamente. 


\section{RESULTADOS}

Una vez efectuado el Análisis Proposicional del Discurso (APD) según los procedimientos establecidos (Ghiglione et al., 1985), se obtuvieron los referentes más importantes, ordenados siguiendo el criterio de su frecuencia de aparición. Estos referentes se presentan y comentan a continuación.

Entrevista (37\%), referente modal, indica que la intención de la entrevista abierta se cumplió, ya que las entrevistadas hablan a partir de su propia experiencia, ideas, sentimientos.

Papalomoyo (23\%), es la designación corriente que usa la población estudiada para referirse a la Leishmaniasis Cutánea; indica que las entrevistas se mantuvieron dentro de la temática predeterminada por la consigna, es decir dentro del interés de la investigación (este término se emplea en lo sucesivo, respetando así las características de las producciones discursivas analizadas).

Chiquitos (19\%), indica que, dentro de la esfera de la experiencia de las entrevistadas, los niños son los más afectados por la Leishmaniasis Cutánea (lo cual coincide con los registros epidemiológicos).

Uno (10\%), indica que la entrevistada generaliza su propia experiencia directa, al mismo tiempo que incorpora la de otras personas que pertenecen a su contexto social.

Lllaga (10\%) es la manifestación clínica de la Leishmaniasis Cutánea, que abre y al mismo tiempo limita la intervención de la entrevistada, en cuanto mujer, con respecto a esta enfermedad.

Medicina casera (9\%), abarca todos los medios de acción directos de la entrevistada sobre el papalomoyo.

Enfermedades (8\%), indica otras preocupaciones relacionadas con la salud, especialmente de los niños, aparte del papalomoyo.

Aliblanco (6\%), es el nombre vernáculo aplicado al género Lutzomya, y a algunas especies de otros géneros. Su rol como vector del papalomoyo parece estar bastante claro en el pensamiento de la gente.

Estos referentes conforma una red cognitiva cuyo centro es el referente entrevistada, que conforma un triángulo cognitivo con los referentes papalomoyo y chiquitos, con los que tiene las relaciones más fuertes. Los otros referentes más frecuentes (excepto enfermedades, que no se conecta con ningún otro) completan el esquema.

El carácter central del referente entrevistada pone de relieve que el conocimiento, las actitudes y la práctica de las entrevistadas sobre el papalomoyo parten de su propia experiencia. Es un discurso centrado en el sujeto, tal como se requería para el estudio propuesto. El referente uno parece ser al menos parcialmente equivalente al referente entrevistada, pero desplazo como para tomar distancia cuanto más el discurso se aleja del papalomoyo en general para tratar aspectos más vivenciales.

La relación más fuerte (entrevistada/ papalomoyo) corresponde al tema de la entrevista, y como se dijo antes indica un alto grado de involucramiento de las entrevistas con la enfermedad. Aquí el referente papalomoyo aparece netamente diferenciado de los referentes llaga (manifestación clínica) y aliblanco (vector), lo que subraya el hecho de que, para las entrevistadas, constituye una entidad nosológica en sí.

La fuerte relación entrevistada/chiquitos establece la esfera de la experiencia en que la enfermedad toma realidad para la entrevistada. Completando y apoyando lo anterior, la relación papalomoyo/chiquitos deja claro que para las entrevistadas la Leishmaniasis Cutánea es, cognitiva y afectivamente, una afección de los niños.

La relación entrevistada/entrevistador(a) es un resultado lógico del contexto y del instrumento de obtención de la información. Se estableció una relación fuerte entre dos personas unidas por una común preocupación por la Leishmaniasis Cutánea.

Entrevistada/llaga localiza y delimita la esfera de acción de la entrevistada sobre la enfermedad: se ejerce sobre la manifestación clínica, a partir del momento en que ésta es evidente. Generalmente no se considera actuar sobre los otros componentes de esta enfermedad A(vector, reservorio, contexto espaciotemporal del contacto).

Entrevistada/medicina casera, por su parte, establece los medios de acción autónoma de la entrevistada sobre le enfermedad, mediante la aplicación de medicamentos caseros de varias clases. Si es exitosa, la entrevistada domina la enfermedad en el doble sentido de que la 
detiene y que no se hace necesario encargar su curación a los médicos.

La relación entre los referentes uno/ papalomoyo es la más débil de todas, por debajo del $5 \%$. Como ya se dijo, extrapola y generaliza la experiencia personal de la entrevistada hacia un sujeto abstracto que comparte sus características sociales. Esta distanciación puede ser motivada por el estigma social asociado al papalomoyo por la población de esas zonas, como indicador de suciedad y pobreza.

Las tendencias que el análisis cuantitativo hizo evidentes guiaron el establecimiento de generalizaciones empíricas a partir de un análisis cualitativo de las entrevistas.

El contexto microsocial de la ocurrencia del papalomoyo es el determinado por la relación entre los referentes entrevistada-papalomoyochiquitos, es decir el contexto hogareño a (que incluye la casa y espacio circundante en un radio de unos 30 metros, donde ella trabaja y los niños juegan). Este ámbito es preponderantemente femenino, su control (aseo, ordenamiento, estética) es de la incumbencia y responsabilidad de la mujer.

Es un ámbito socializado, culturizado, en el cual el aspecto caótico de la naturaleza está, justamente, domesticado. A las mujeres les corresponde el cuido y el aseo de los animales que ahí se encuentran (cerdos, gallinas, patos, perros), el escogimiento y el cuido de las plantas (decorativas y medicinales principalmente; prácticamente no hay plantas alimenticias excepto frutales). En ese sentido, el tener plantas decorativas (de colores vivos, variadas, bien mantenidas) es un aspecto muy importante para ellas, porque consideran que "una casa sin flores es una casa sin mujer".

Las personas entrevistadas no tienen conciencia clara del posible rol de los animales ni de las plantas que se encuentran en ese ámbito en el aumento o disminución del riesgo de transmisión del papalomoyo; sin embargo a algunas de esas plantas se les considera importantes como fuente de sustancias curativas para esta afección (limón ácido, por ejemplo).

La ocurrencia del papalomoyo representa una irrupción del desorden de la naturaleza en el ámbito hogareño, idealmente seguro (tanto socialmente como sanitariamente), y que las mujeres asumen la responsabilidad de controlar.
En ese esfuerzo están apoyadas por sus esposos; éstos están encargados de conseguir algunos de los elementos necesarios para su curación hogareña, si no se encuentran en el ámbito peridoméstico (juntar savia del árbol targuá, cazar tepezcuintle para extraerle la hiel, por ejemplo). Asimismo, recurren a personas mayores, hombres o mujeres (padre, madre, tío, suegra, vecino, conocido), detentoras de un saber tradicional transgeneracional, para confirmar el diagnóstico o para confirmar, recomendar o proponer alternativas de tratamiento.

Se pudo notar, a través de comentarios y juicios sueltos en las entrevistas, que el papalomoyo tiene una connotación de estigma social, en términos de suciedad, descuido, descontrol, desorden y pobreza. Esta estigmatización puede ligarse con lo comentado más arriba: la ocurrencia del papalomoyo, al marcar el ingreso de la naturaleza y su desorden en el ámbito hogareño, sugiere una falta o pérdida de control sobre la casa, sobre el mundo socializado que ésta representa (descontrol social).

El estigma afecta en primer lugar a la mujer, por lo que se ha dicho, pero también al hombre, por cuanto se estima que no pudo o no supo proveer los medios necesarios para que ella asegurara el debido control (una casa lo más cerrada posible, letrinas en buen estado, desagües, encierros para los animales); los niños no están sujetos a él puesto que no se les responsabiliza de esas situaciones (posiblemente es por eso que se habla libre y recurrentemente del papalomoyo en niños y poco espontáneamente del papalomoyo en adultos).

Generalmente la idea de que la transmisión pudo haber ocurrido en el ámbito hogareño no es fácilmente aceptada por las entrevistadas, que a lo sumo sugieren que pudo haber sucedido por la noche (dando a entender que en ese momento si control sobre este ámbito disminuye). Tienden más bien a buscarle explicación en algún desplazamiento a un lugar ajeno.

Recurrentemente, el lugar presumido de transmisión se ve caracterizado como lejano, más "húmedo" o más "caliente" que el lugar de residencia. Suele asociarse a barreales y char$\cos$ ("suciedad" natural) y más específicamente a coberturas boscosas ("montañillas") o a corrientes de agua (experiencia empírica con- 
vergente con el ciclo selvático de la enfermedad, determinado por la epidemiología anterior a 1970 , pero no con las nuevas condiciones de transmisión peridoméstica o intradoméstica).

El control del papalomoyo por parte de las mujeres, y con los medios a su disposición, constituye un intento de reponer orden en el ámbito hogareño. El componente de la enfermedad que se encuentra al alcance de la mujer, y que por lo tanto circunscribe su acción, es la llaga. Se puede afirmar que, para las entrevistadas, la remisión de la llaga equivale a la curación de la enfermedad (aunque se tiene por preferible llegarle "a la raíz"; este ente causal se considera localizado en la llaga).

La conciencia de la mediación del aliblanco, insecto volador hematófago, vector en el proceso de transmisión de la enfermedad, está bastante extendida. Sin embargo no se menciona nunca la posibilidad de controlarlo, sobre todo porque no se visualiza que esa mediación pueda darse en el ámbito hogareño, y en consecuencia no consideran que evitarla se a de su incumbencia.

Tampoco se menciona la posibilidad de controlar otros componentes de la enfermedad (por ejemplo los reservorios). Esto se explica en parte porque las entrevistadas no tienen muy bien definidos esos componentes, pero sobre todo porque, como queda claro, la idea de que pudiera existir transmisión de la enfermedad en el espacio doméstico no resulta aceptable para la mayoría.

Es también la llaga la que da pie para el diagnóstico del papalomoyo: éste se declara cuando las manifestaciones tempranas no respondieron a su tratamiento como simples granos. Si no es grano, es probablemente papalomoyo (suele recurrirse a otras opiniones, preferiblemente de personas mayores, para conformarse en un diagnóstico que de hecho ya se ha establecido). Por otra parte, el tiempo durante el cual se le trata como a un grano cualquiera, también corresponde al necesario para que la lesión adquiera su aspecto más típico, cuyas características están definidas con bastante precisión.

En efecto, popularmente se reconocen claramente dos tipos de papalomoyo. El primer tipo tiene dos formas: "seco" (cubierto por una "cascarilla", se extiende muy rápidamente pero superficialmente, carcomiendo por los bordes de la lesión); "ciego" (también cubierto por una "cascarilla" y superficial, pero no evoluciona). En contraposición, se reconoce un segundo tipo de Leishmaniasis Cutánea, que suscita más discurso: el "de cacho o cachillo", también llamado "aguado" o "llorado" (secreta líquido, carcome la piel pero hacia adentro, "buscando la carne", en forma de "remolino").

A veces se tratan las lesiones metastatizadas como un tercer tipo: los papalomoyo "con hijitos”. Ningún informador explicitó más que los tipos mencionados, aunque se afirma de manera recurrente que "los mayores llegaban a reconocer hasta treinta clases".

Independientemente de su clasificación, al referirse a las llagas se dice de ellas que "duelen", "arden”, "punzan", "hierven la piel”, "aquello se pudre". Los papalomoyos "aguados", y también los "secos" en su segunda fase de evolución, se describen como que producen líquidos amarillentos, blancuzcos, morados, rojos (suero, sangre, pus).

Al hablar de las lesiones se hace referencia implícita a un ente patógeno localizado en ellas (congruentemente con el saber parasitológico); se le atribuyen rasgos humanizantes (agresividad, voluntad, inteligencia, decisión): "muerde", "punza", "come", "se siente acorralado y entonces ataca con más fuerza", "se defiende", "el mandamás tira hijitos", "el demonio se jarta", "los hijitos son más bravos que el papá", "se quiere amortiguar". Cuando se ejerce la acción de curar mediante medicina casera, importa "secar" la lesión; sin embargo su fin último es "matar" a este ente patógeno.

Los productos vegetales, animales y manufacturados utilizados con fines terapéuticos (Cuadro 1). Todos esos medicamentos caseros se aplican directamente sobre la llaga, unos sin preparación previa aparte del hecho de haber sido obtenidos fuera del ámbito casero y traídos a él, otros después de preparación previa. Se caracteriza por compartir uno o más de los atributos con los que se define a la llaga: queman, destruyen la piel o son líquidos amarillentos, blancuzcos, rojos, o provienen de una herida punzante ("chuzazo"). Por otro lado, por una u otra razón intrínseca a cada uno y, en distintas medidas, son considerados venenosos o peligrosos en sí. 
CUADRO 1. Productos Utilizados como Medicamentos Caseros Tradicionales en el Cantón de Acosta

\section{Vegetales}

- Manchas (latex) de árbol, extraídas de su tronco:

- ojoche (Brosinum costaricanum Liebm. y B. terrabanum Pittier)

- achiotillo (Vismia guiaanensis (Aubl.) Pers.)

- targuá (Cortón gossypiifolius Vaahl.)

- higuerón (Ficus sp.)

- jinocuabo (Bursera Simarubaa (L.) Sarg.)

- Manchas de árbol, obtenidas al arrancar su fruta:

- huevo de caballo o guijarro (Stemmadenia Alfari, S. obovata)

- Preparaciones de:

- jugo de limón ácido caliente (Cirus aurantifolia)

- mezcla de 7 hojas y 7 frutas celes de guayabo (Psidium guajava)

- resina de tabaco "derretido" (miel) de pipa (Nicotiana tabacum L.)

- Otros:

- hojas y frutas venenosas (sin determinar)

- humillo de la cáscara de la naranja (Citrus sinensis L.)

- carbón, tizne

\section{Animales}

- Productos de aplicación directa:

- garrapata (Ixodides) arrancadas a perros o ganado

- boñiga de vaca muy fresca

- mantequila de leche agriada

- hiel de tepezcuintle (Cuniculus paca)

- heces de recién nacido

- yema de un huevo de gallina (Gallus gaallus) hervido durante varias horas

\section{Industrializados}

- Pomadas a base de manteca (de cerdo o industrializada):

- suero y ácido bórico

- yodo con ácido bórico

- sulfatiasol

- compuesto de ácido bórico con ácido sulfúrico (proveniente de una batería de carro descompuesta).

- Líquidos

- líquido de inyecciones de glucantime en forma tópica

- alcohol con alcanfor

- alcohol puro

- agua florida

- Otros:

- betún negro

- esperma de candela caliente 
En general, pues, se responde al principio de curar "lo mismo con lo mismo", según la concepción que se tiene tanto de la llaga como de la sustancia curativa: la utilización de "venenos" en este contexto provee una indicación sobre la concepción que se tiene del ente patógeno y de si manera de actuar dentro de la llaga: es venenoso, ponzoñoso.

Esto se ve confirmado por una idea común entre las entrevistadas, según la cual ese veneno lo transportaría un insecto desde un sapo o una culebra venenosos, a veces muertos. En cuanto al "veneno" usado para curar el papalomoyo, se trata de un "mismo" modificado, socializado por el manejo que la ha dado la mujer, que es lo que posibilita su eficacia.

En contraposición a la cura en el ámbito hogareño, las mujeres tienen la posibilidad de acudir a tratamientos médicos formales (sobre todo en los últimos años, en que un programa específico ha venido extendido y haciendo efectiva esa oferta en el cantón de Acosta).

Sin embargo se ha constatado que las lesiones que se tratan en esta consulta suelen estar avanzadas; eso confirma lo que resalta del análisis de las entrevistas, a saber que esta alternativa sólo se escoge en última instancia, después de haber recurrido a la medicina casera y en los casos en que su aplicación no resultó exitosa en un plazo considerado por las mujeres como razonablemente corto.

Si bien es cierto que para las mujeres el desplazarse para acudir a esos tratamientos médicos formales implica algún esfuerzo económico, de tiempo y hasta físico, sobre todo implica salirse del ámbito hogareño que ellas controlan. En efecto, el ingresar a este otro ámbito más amplio, defensor de la salud también, significa para ellas ceder el control sobre la salud de las personas, hasta entonces exclusivamente a su cargo: esta situación afectiva podría explicar aún mejor la reticencia y la tardanza en acudir a los servicios médicos disponibles.

\section{DISCUSIÓN Y CONCLUSIONES}

Los resultados del presente estudio apuntan hacia algunas conclusiones importantes, en relación al estudio epidemiológico con el cual está ligado y en relación al diseño de un pro- grama de control experimental que deriva de ambos y que tiene como principio el ser compatible con las características sociales y culturales de la población a riesgo (condición necesaria para su eficaz aplicación).

El análisis epidemiológico ha establecido que existe un importante riesgo de transmisión de Leishmaniasis Cutánea en el espacio doméstico y peridoméstico; el análisis antropológico muestra que este mismo espacio es considerado por parte de la población estudiada como el que ofrece la mayor seguridad al respecto. Este estudio muestra que las mujeres, que son culturalmente y socialmente responsables del ámbito doméstico y peridoméstico, no tienen conciencia clara de que varios factores de la enfermedad se encuentran dentro de ese espacio.

Un programa de educación bien dirigido debería hacer evidente que el espacio doméstico y peridoméstico es de hecho riesgo. Bajo esa condición se puede prever una actitud positiva por parte de los miembros de la comunidad y especialmente de las mujeres, para la aplicación de medidas de control dirigidas a incrementar la seguridad (sanitaria, en este caso) del espacio doméstico y peridoméstico.

La campaña de control debería ofrecer una información amplia respecto al funcionamiento de la Leishmaniasis Cutánea, indicando y favoreciendo medidas de control casero de esos factores. Tal control debería prioritariamente depositarse en manos de las mujeres amas de casa, respetando así su rol tradicional de responsables del espacio hogareño.

El análisis epidemiológico ha establecido, por otra parte, que algunas plantas y animales domésticos que se encuentran en el ámbito hogareño pueden tentar una función de aumento o de disminución del riesgo que corre la población de adquirir Leishmaniasis Cutánea; sin embargo el presente estudio antropológico hace evidente que la población no concibe relación alguna entre aquellos y la enfermedad (si no es indirecta y vagamente, porque pueden implicar un aumento de "suciedad").

Es probable que modificar las condiciones de existencia de los animales domésticos dentro del ámbito casero (construir un chiquero o alejarlo de la casa si ya existe; encerrar las gallinas en gallinero, con el incremento de 
cuido y los costos asociados) encontraría una cierta resistencia por parte de la población, que concibe que deben desplazarse libremente. A eso se debe posiblemente la mala acogida que encontró una reciente campaña que, frente al riesgo de un brote de cólera, internó eliminar o encerrar indiscriminadamente esos animales.

Aunque en principio el control de la enfermedad puede dirigirse a varios de sus componentes (llaga, vector, reservorios, huéspedes, lugares, épocas), este estudio mostró que las mujeres, que son en la práctica las encargadas de su control primario, circunscriben conceptualmente y prácticamente el alcance de su acción a la llaga, procurando eliminar el ente venenoso que conciben se encuentra en ésta.

El programa de control debería pues seguir dando importancia al tratamiento de la llaga, en el tanto en que su cura constituye la principal relación de la población con la Leishmaniasis Cutánea, así como su principal motivación.

Este estudio exploratorio constituye un ejemplo ilustrativo de cómo los resultados cualitativos de la disciplina antropológica pueden ponderar los resultados cuantitativos de la disciplina epidemiológica y favorecer la aplicación de medidas de control dirigidas a reducir la incidencia de una enfermedad.

\section{RESUMEN}

DOBLES-ULLOA, A. \& PERRIARD, C.

Representaciones, Actitudes y Prácticas Respecto a la Leishmaniasis Cutánea en la Población del Cantón de Acosta, Provincia de San José, Costa Rica. Estudio Antropológico Exploraatorio. Cad. Saúde Públ., Rio de Janeiro, 10 (2): 181-189, abr/jun, 1994.

Estudio antropológico exploratorio sobre las representaciones, actitudes y prácticas relacionadas con la Leishmaniasis Cutánea (LC) en la población rural del cantón de Acosta, Costa Rica, dirigido a estimar la posibilidad de aplicación de medidas de control propuestas sobre una base epidemiológica. Entrevistas abiertas con una pequeña muestra de personas, provenientes tanto de casas-caso como de ca- sas-control proveyeron la base para un Análisis Proposicional del Discurso (APD). Los resultados son que la gente de Acosta considera la LC como una entidad nosológica diferenciada, pero se interesan sobre todo en sus manifestaciones clínicas en los niños (que son los más afectados), así como en su propia capacidad de acción mediante remedios populares. La idea de medidas de control sobre los reservorios, los vectores o el contexto espaciotemporal del contacto no asoma espontáneamente en el pensamiento de la gente. Sin embargo, la LC se percibe como una disrupción en el espacio doméstico y peridoméstico, considerado seguro; medidas de control que intervengan en ese ámbito podrían pues tener buenas posibilidades de éxito.

Palabras Clave: Leishmaniasis; Antropología; Control; Tratamiento; Epidemiología

\section{REFERENCIAS}

DEVEREUX, G., 1970. Essais D'Ethnopsychiatrie Générale. Paris: Gallimard.

GHIGLIONE, R.; MATALONE, B. \& BATRI, N., 1985. Les Dires analysés. L'Analyse propositionnelle du Discours. Saint-Denis: Preses Universitaires de Vincennes.

MURILLO, J. \& ZELEDÓN, R., 1985. Flebótomos de Costa Rica (Diptera: Psychodidae). Brenesia, 23: 1-137.

NUHN, H., 1978. Atlas Preliminar de Costa Rica. San José: Instituto Geográfico Nacional.

ROJAS, J. C., 1992. New strategy for the control of Cutaneous Leishmaniasis: the case of Acosta, Costa Rica. In: Leishmaniasis Control Strategies: A Cristal Evaluation of IDRCsupported research (P. Wijeyaratne; T. Goodman \& C. Espinal, eds.), pp. 223-229, Ottawa: International Development Research Centre.

ROJAS, J. C., ZELEDON, R.; MURILLO, J. \& URBINA, A., 1988. Identification of risk factors associated with Cutaneous Leishmaniasis in Costa Rica. In: Research on Control Strategies for the Leishmaniases (B. C. Walton; P. M. Wijeyaratne \& F. Modabber, eds.), pp. 244-251, Ottawa: International Development Research Centre. 